University of Windsor

Scholarship at UWindsor

7-2019

\title{
Concentration of Bacterial Specimens During Centrifugation Prior to Laser-Induced Breakdown Spectroscopy Analysis
}

\author{
Alexandra E. Paulicka \\ University of Windsor \\ Dylan J. Malenfanta \\ University of Windsor \\ Steven J. Rehse \\ University of Windsor
}

Follow this and additional works at: https://scholar.uwindsor.ca/physicspub

Part of the Physics Commons

\section{Recommended Citation}

Paulicka, Alexandra E.; Malenfanta, Dylan J.; and Rehse, Steven J.. (2019). Concentration of Bacterial Specimens During Centrifugation Prior to Laser-Induced Breakdown Spectroscopy Analysis.

Spectrochimica Acta Part B: Atomic Spectroscopy, 157, 68-75.

https://scholar.uwindsor.ca/physicspub/196

This Article is brought to you for free and open access by the Department of Physics at Scholarship at UWindsor. It has been accepted for inclusion in Physics Publications by an authorized administrator of Scholarship at UWindsor. For more information, please contact scholarship@uwindsor.ca. 
Concentration of Bacterial Specimens During Centrifugation Prior to Laser-Induced Breakdown Spectroscopy Analysis

Alexandra E. Paulicka, Dylan J. Malenfant ${ }^{\mathrm{a}}$, Steven J. Rehse ${ }^{\mathrm{a},{ }^{*}}$

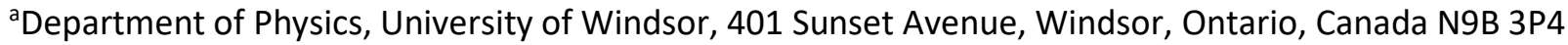

*Corresponding author at: Department of Physics, University of Windsor, 401 Sunset Avenue, Windsor, Ontario, Canada N9B 3P4. Tel.: +1 5192533000

E-mail address: rehse@uwindsor.ca (S.J. Rehse).

\section{HIGHLIGHTS}

- Bacteria are readily trapped on sub-micron pore size filter media.

- Centrifuging bacterial suspensions easily collects cells on removable filters.

- A metal cone concentrates bacterial cells in an area less than $1 \mathrm{~mm}$ in diameter.

- The limit-of-detection was reduced to approximately 11,000 cells per laser shot.
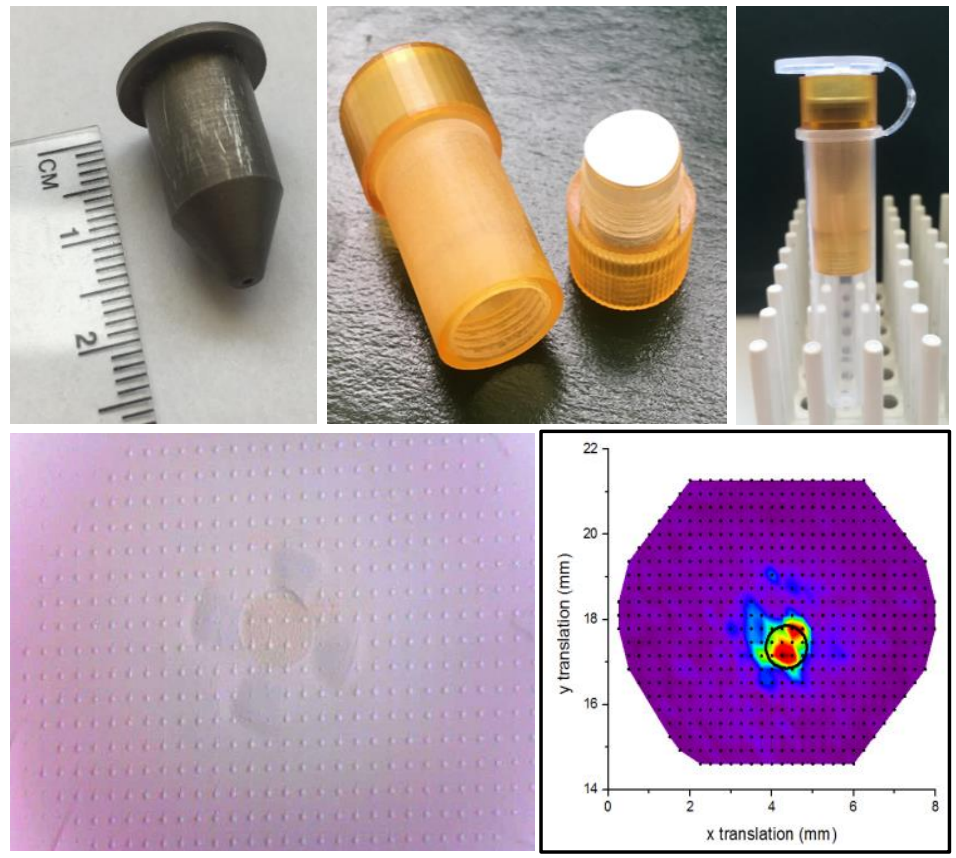


\section{ABSTRACT}

A metal cone device has been designed and fabricated for use with a custom centrifuge tube insert to allow the simple and rapid concentration of bacterial cells in a circular area with a diameter of $1 \mathrm{~mm}$. The device concentrates cells suspended in up to $1 \mathrm{~mL}$ of liquid at the center of a highly flat disposable filter medium which can be easily removed from the centrifuge tube insert for subsequent testing with laserinduced breakdown spectroscopy. Two-dimensional elemental mapping of the filter evidenced a high concentration of bacteria on the filter under the location of the cone hole, as well as detection of reduced bacterial signal in peripheral areas slightly outside the footprint of the cone hole indicating some leakage of bacteria. Nine different dilutions of a suspension of $E$. coli were prepared and after measurement of the titer by optical densitometry, deposited on the filter media using the cone concentrator. A calibration curve constructed from forty spectra obtained from each of the nine different concentrations returned a LIBS bacterial limit of detection of 10,865 $\pm 3,712$ CFU per laser ablation event for bacteria deposited on filters using the metal cone. Limits of detection calculated using only certain elements observed in the LIBS spectra and present in very low concentrations in the filter were even lower: 1,070 $\pm 272 \mathrm{CFU}$ for magnesium and 1,784 $\pm 657 \mathrm{CFU}$ for calcium. This represents a factor of 50 reduction in the limit of detection compared to our previously reported value.

\section{KEYWORDS}

Laser-induced breakdown spectroscopy; Bacteria concentration; Centrifugation; Filter 


\section{Introduction}

The use of the spectroscopic technique known as laser-induced breakdown spectroscopy (LIBS) for a variety of important medical, clinical, and biomedical applications has been welldocumented $[1,2,3,4]$. Of particular importance in this area of application is the rapid detection and subsequent identification of infectious pathogens in clinical specimens or in the environment. The LIBS spectra from pathogenic micro-organisms such as fungal spores and yeast have been obtained and the suitability of these spectra for differentiating those pathogens from other microorganisms has been investigated $[5,6,7,8]$. In addition, the use of LIBS for virus identification, while not well-studied, has been demonstrated $[9,10]$.

The use of LIBS for bacterial detection and identification has been studied to a significantly greater extent than all the other pathogens, as evidenced by the number of publications that have appeared on the topic over the past fifteen years [11]. This work has consistently demonstrated the ability of LIBS to differentiate or discriminate the LIBS spectra obtained from bacterial cells due to the uniqueness of the elemental composition of different bacterial genera and species $[12,13,14]$. This uniqueness extends to subtle difference between strains of a species that can be reliably detected in the LIBS spectra $[15,16,17,18]$.

Given the sensitivity and specificity demonstrated in these previously described works, it is clear the use of LIBS for rapid bacterial detection or identification could be an important tool for the clinical microbiologist if the calculated performance metrics persisted for the low bacterial cell count (titer) anticipated in clinical specimens and not merely for tests performed on arbitrarily, but unrealistically large, numbers or concentrations of cells $[19,20,21]$. As such, the quantification of the limit-of-detection (LOD) or the limit-of-identification (LOI) of the technique, and the demonstration of efficacy on specimens with clinical titers, is paramount. Ideally, a LIBS spectrum which could be used to detect and correctly identify unknown pathogenic bacteria could be obtained from specimens with a concentration of 1-100 colony forming units (CFU)/mL (the concentration of microbes in a typical blood sample from a bacteremic patient [22]) or 
specimens containing an absolute cell count in the range of 0-200 CFU (the number of cells of methicillin-resistant S. aureus (MRSA) typically recovered from a nasal swab of an infected patient [23]).

While spectra from single cells or spores have been obtained, the methods used to generate these spectra do not readily recommend themselves for use in a clinical environment [7]. Other studies have investigated the LOD by performing sequential experiments on serial dilutions of bacterial suspensions or preparations of various bacterial titers. Typical LODs of around $10^{5}-10^{6}$ $\mathrm{CFU} / \mathrm{mL}$ have been found [24,25], although some studies have shown good identification with concentrations in the range of $10^{3}-10^{4} \mathrm{CFU} / \mathrm{mL}[26,27]$ and at least one study has shown efficient discrimination of two bacterial species for all concentrations in the range of $10^{0}-10^{5}$ $\mathrm{CFU} / \mathrm{mL}[28]$.

In this paper we present a description of a rapid and efficient technique for concentrating bacterial cells suspended in a liquid medium upon inexpensive disposable filtering medium. The technique utilizes a custom fabricated centrifuge-tube insert and cone device that is used during centrifugation of the liquid specimen. The entire assembly and process has been designed to be rapid, safe, and performable by laboratory personnel with little specialized training and requiring little specialized apparatus. In this way we were able to filter sizeable volumes of bacteriacontaining liquid (in this case water, but equally applicable to blood, urine, cerebral spinal fluid, etc.) and create bacterial depositions that possessed enough cells to fall within the anticipated limits of detection as reported previously in the literature. 


\section{Material and Methods}

\subsection{LIBS apparatus}

The LIBS apparatus used in this work has been described elsewhere [25]. It consisted of a 1064 $\mathrm{nm} \mathrm{Nd:} \mathrm{YAG} \mathrm{laser} \mathrm{operating} \mathrm{at} 10 \mathrm{~Hz}$ with a pulse duration of approximately $10 \mathrm{~ns}$ and a pulse energy of $8 \mathrm{~mJ}$ focused to a circular spot approximately $75 \mu \mathrm{m}$ in diameter utilizing a long working distance AR-coated microscope objective. Ablation was performed within a purged Plexiglas ablation chamber to enable ablation of the target in a controlled gas environment. All work described in this manuscript was performed in an argon over-pressure environment by maintaining an argon flow of 20 SCFH during data acquisition.

The chamber possessed a manual translation stage allowing translation of the target in three dimensions, ensuring sampling of the target at different locations and efficient focusing onto the surface of the target. All targets ablated in this work were thin lawns of Escherichia coli (E. coli) deposited on circular $13 \mathrm{~mm}$ diameter disposable nitrocellulose filter papers with a pore size of $0.45 \mu \mathrm{m}$ (HAWP01300, Millipore-Sigma) which were mounted on a steel piece using double-sided tape. Prior to any testing, all filters were trimmed to an outer diameter of $9.5 \mathrm{~mm}$ to allow them to fit within the centrifuge tube insert.

Emission from the LIBS plasmas in the range $200-840 \mathrm{~nm}$ was collected by a pair of matched parabolic reflectors and directed into an Echelle spectrometer (ESA 3000, LLA instruments GmbH) by an optical fiber. The Echelle spectrometer was equipped with an intensified charge coupled device (ICCD) for time gating of the plasma observation. A personal computer running manufacturer-provided software (ESAWIN v3.20, LLA Instruments GmbH) was used to control the gating of the ICCD with respect to the firing of the laser pulses. Plasma emission was collected at a delay time of $2 \mu$ s after plasma formation with a gate window of $20 \mu \mathrm{s}$. In all cases, a single laser shot was sufficient to remove all the bacteria illuminated by the laser pulse. This removal was verified both spectroscopically - subsequent laser pulses yielded spectra that were not 
distinguishable from blank filter spectra - as well as by scanning electron microscopy and highresolution optical microscopy. The focal spot was translated $0.25 \mathrm{~mm}$ to expose a new surface after every laser shot. Single laser shots provided adequate signal-to-noise for quantification and spatial mapping, and occasionally three single-shot spectra were digitally averaged to minimize shot noise. Fig. 1(a) shows three representative LIBS spectra acquired in this study for two concentrations of $E$. coli and the blank filter upon which they were deposited. The blank filter spectrum is the average of 20 individual single-shot spectra and the two $E$. coli spectra are the average of 40 individual single-shot spectra to improve the signal-to-noise. The spectra have been overlaid to illustrate the relative strengths of the LIBS emissions. To better highlight the difference observed in measured emission lines, zoomed-in details of the spectra surrounding emission lines in phosphorus (253.560 nm), magnesium (279.553, 279.806, and $280.271 \mathrm{~nm})$, and calcium (393.366 and $396.874 \mathrm{~nm}$ ) are shown in Fig. 1(b), 1(c), and 1(d), respectively.

A total of 19 emission lines from carbon, phosphorus, magnesium, calcium, and sodium were used in the analysis of bacterial spectra. The background-subtracted integrated area-under-thecurve intensities of these 19 emission lines were measured by the ESAWIN software. The sum of the intensities of the 19 emission lines represented the total LIBS intensity for a single spectrum, which is proportional to the number of cells deposited on the filter. Table 1 lists the 19 emission lines used in this work and also the averaged values (in arbitrary units) obtained by measuring 40 spectra from two representative $E$. coli concentrations and 20 spectra from a blank nitrocellulose filter upon which no bacteria were deposited. 

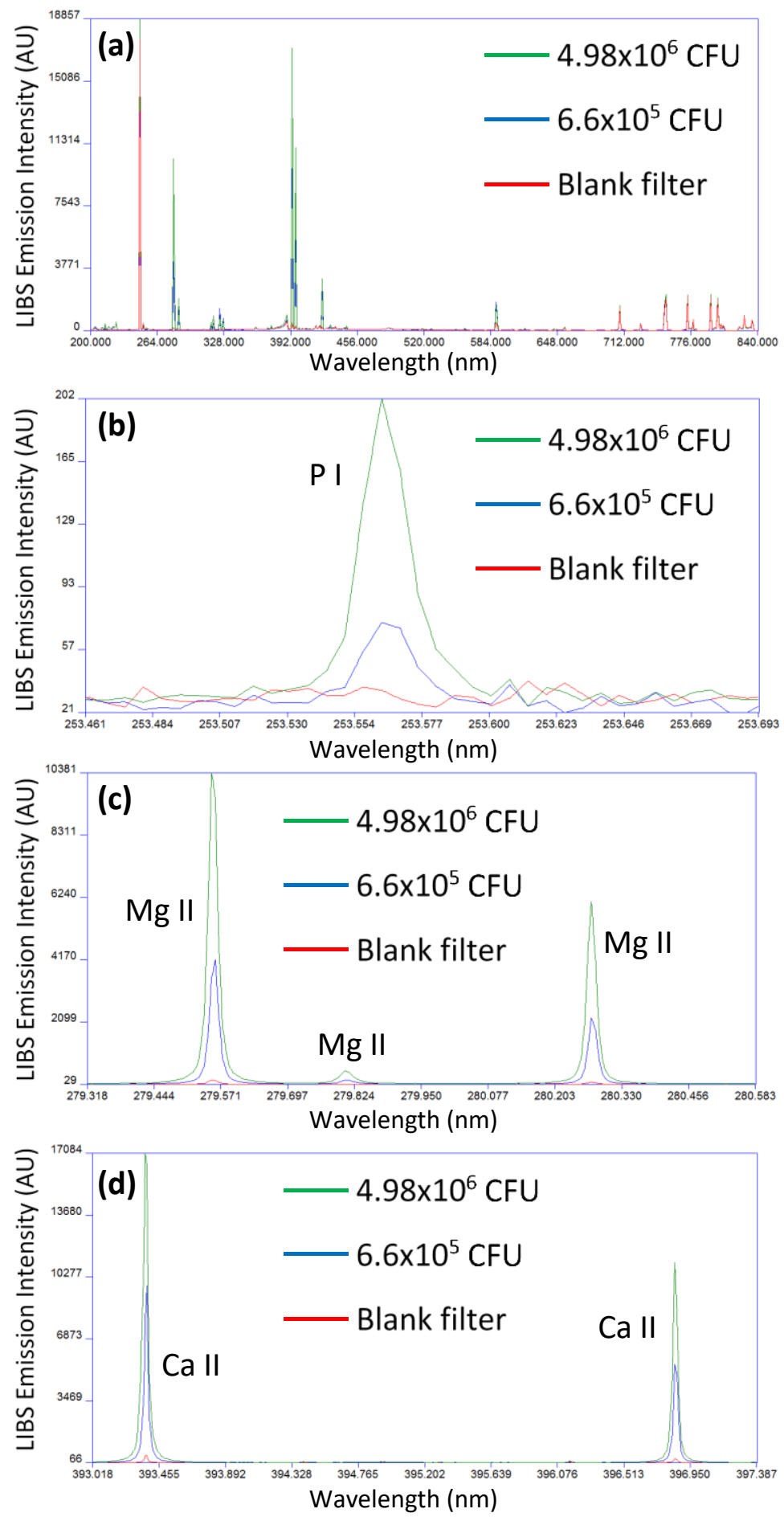

Fig. 1. (a) Overlay of three LIBS spectra, each the average of 40 single-shot LIBS spectra (for E. coli) or 20 single-shot spectra (for the blank filter) acquired with the same parameters. Spectra were acquired from two concentrations of E. coli bacteria: $4.98 \times 10^{6}$ CFU (green) and $6.6 \times 10^{5} \mathrm{CFU}$ (blue) and a blank nitrocellulose filter upon which all the bacteria were deposited (red). (b) $A$ zoomed in detail of the spectra in the region of the P I emission line at $253.560 \mathrm{~nm}$. (c) A zoomed in detail of the spectra in the region of three $\mathrm{Mg}$ II emission lines at 279.553, 279.806, and $280.271 \mathrm{~nm}$. (d) A zoomed in detail of the spectra in the region of two Ca II emission lines at 393.366 and $396.874 \mathrm{~nm}$. 
Table 1

Identification of the nineteen LIBS emission lines used for bacterial limit of detection measurements and averaged values for two representative bacterial titers and a blank nitrocellulose filter.

\begin{tabular}{|c|c|c|c|c|}
\hline Element & $\begin{array}{l}\text { Wavelength } \\
(\mathrm{nm})\end{array}$ & $\begin{array}{c}\text { Average LIBS Emission } \\
\text { Intensity (AU) - Bacteria } \\
4.98 \times 10^{6} \mathrm{CFU}\end{array}$ & $\begin{array}{c}\text { Average LIBS Emission } \\
\text { Intensity (AU) - Bacteria } \\
6.6 \times 10^{5} \mathrm{CFU}\end{array}$ & $\begin{array}{c}\text { Average LIBS Emission } \\
\text { Intensity (AU) - } \\
\text { Blank Filter }\end{array}$ \\
\hline $\mathrm{C} \mathrm{I}$ & 247.856 & 40342 & 37365 & 38082 \\
\hline P I & 213.618 & 1649 & 1428 & 83 \\
\hline P I & 214.914 & 879 & 391 & 98 \\
\hline P I & 253.398 & 81 & 46 & 33 \\
\hline P I & 253.560 & 387 & 128 & 46 \\
\hline P I & 255.326 & 146 & 65 & 35 \\
\hline P I & 255.491 & 79 & 45 & 37 \\
\hline $\mathrm{Mg}$ II & 279.079 & 722 & 196 & 37 \\
\hline $\mathrm{Mg}$ II & 279.553 & 23476 & 8672 & 318 \\
\hline $\mathrm{Mg}$ II & 279.806 & 1306 & 441 & 34 \\
\hline $\mathrm{Mg}$ II & 280.271 & 12769 & 4613 & 163 \\
\hline Mg I & 277.983 & 141 & 80 & 42 \\
\hline Mg I & 285.213 & 3343 & 1918 & 112 \\
\hline $\mathrm{Ca}$ II & 317.933 & 3698 & 1409 & 61 \\
\hline $\mathrm{Ca}$ II & 393.366 & 41316 & 17735 & 722 \\
\hline Ca II & 396.847 & 19527 & 9144 & 380 \\
\hline $\mathrm{Ca} \mathrm{I}$ & 422.673 & 4275 & 3330 & 237 \\
\hline $\mathrm{Na} \mathrm{I}$ & 588.995 & 2751 & 2699 & 837 \\
\hline $\mathrm{Na} \mathrm{I}$ & 589.593 & 1829 & 1763 & 572 \\
\hline
\end{tabular}




\subsection{Metal cone and centrifuge insert}

The apparatus used for centrifugation and concentration is shown in Fig. 2 and details of its construction have been described elsewhere [29,30]. A $40 \mathrm{~mm}$ long centrifuge tube insert capable of holding and filtering at least $1.5 \mathrm{~mL}$ of liquid was designed and fabricated via 3-D printing. This centrifuge tube insert was designed to fit inside a standard $10 \mathrm{~mL}$ capacity centrifuge tube equipped with a hinged plastic cap (specifically the filtrate collection tube from an Ultrafree-CL centrifugal filter device, Millipore). The lightweight composite insert consisted of a cylindrical tube-shaped main body with a $14 \mathrm{~mm}$ outer diameter. The base of the insert served as a platform upon which the filter was placed (shown in Fig. 2(a)). Screwing the base securely into the main body created a seal around the edge of the filter through applied pressure. The top of the main body was slightly wider at $17 \mathrm{~mm}$, allowing it to rest on the lip of the centrifuge tube without falling to the bottom, shown in Fig. 2(c) and 2(d).
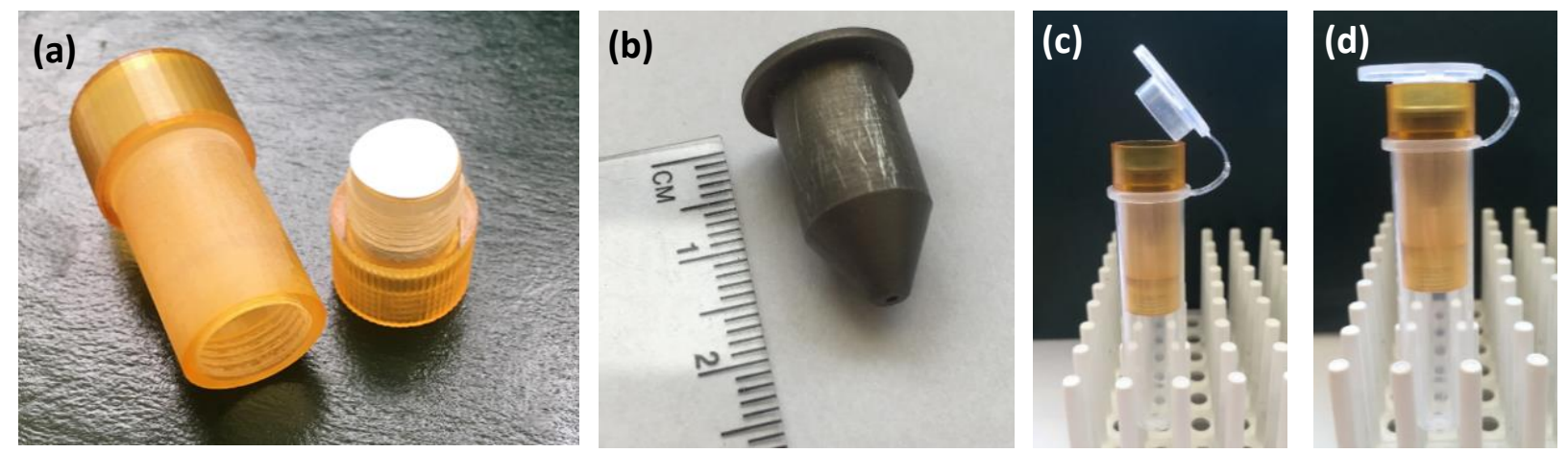

Fig. 2. (a) Disassembled centrifuge insert with filter paper placed on the base. (b) The metal cone prior to being placed in the insert. (c) The metal cone inside the insert which is inside a standard $10 \mathrm{~mL}$ centrifuge tube. (d) Cap of the centrifuge tube presses the metal cone firmly into the filter paper sitting on the base of the insert.

A lightweight hollow metal cone was designed and constructed to fit inside this centrifuge tube insert, forcing the bacterial suspension to pass through the $1 \mathrm{~mm}$ diameter hole at its apex during centrifugation (Fig. 2(b)). The $19 \mathrm{~mm}$ long metal cone was designed to sit on a lip at the top of the centrifuge tube insert and was held securely in place by the centrifuge tube cap, shown lifted in Fig. 2(c) and in place in Fig. 2(d). When the insert base was screwed into the main body of the insert, the filter was pressed firmly into the apex of the cone, forcing the bacterial cells to settle 
onto a region of the nitrocellulose filter approximately the size of the $1 \mathrm{~mm}$ diameter hole as the liquid was pulled through the filter during centrifugation. The cone could hold approximately 1 $\mathrm{mL}$ of bacterial suspension and all samples were centrifuged for 5 minutes at 5,000 rpm $(2,500$ g's of force), sufficient to pull all the liquid through the filter. In these experiments, a Unico PowerSpin BX centrifuge was used, but this cone and centrifuge insert are compatible with any centrifuge equipped with a $15 \mathrm{~mL}$ rotor. After centrifugation, the apparatus was disassembled to remove the filter which was then mounted to a $25 \mathrm{~mm} \times 25 \mathrm{~mm}$ steel piece using double-sided tape and tested with LIBS.

\subsection{Bacterial growth and preparation}

Non-pathogenic Escherichia coli was used for all experiments described in this work. E. coli from a mother culture was inoculated on the surface of tryptic soy agar (TSA) nutrient media, incubated at $37^{\circ} \mathrm{C}$, harvested as colonies from the surface of the growth plates 24-72 hours after streaking, then suspended in $1.5 \mathrm{~mL}$ of deionized water for storage. The bacterial suspensions were stored in a refrigerator until they were required for an experiment. Prior to any experiment, the suspension was vortex-agitated to distribute the cells evenly throughout the suspension. The concentration in CFU/mL for each suspension was determined through optical densitometry measurements on representative volumes of the suspension.

\section{Results and Discussion}

\subsection{Distribution of deposition on filter}

To test the distribution of bacterial cells deposited on a filter after centrifugation through the metal cone, $50 \mu \mathrm{L}$ of an E. coli suspension with a concentration of $9.2 \times 10^{7} \mathrm{CFU} / \mathrm{mL}$ was centrifuged through the metal cone with the conditions described in section 2.2. Single-shot LIBS spectra were acquired across the surface of the filter. Fig. 3(a) shows a false-color map of the total LIBS intensity (in arbitrary units) as a function of position on the filter. The color represents the total measured LIBS intensity as defined in section 2.1. This intensity is the bacterial signal, 
where purple corresponds to no bacterial signal and red corresponds to strong bacterial signal. Purple regions where no bacterial signal was observed are dominated by carbon emission due to ablation of the nitrocellulose filter medium. Each black dot represents a single laser shot and the superimposed black circle ( $1 \mathrm{~mm}$ in diameter) shows the approximate location of where the cone was pressed into the filter. Fig. 3(b) shows an optical micrograph of the filter taken after data acquisition. The array of craters due to the stepping of the laser shots are visible across the filter. The $1 \mathrm{~mm}$ diameter region of bacterial deposition at the center of the filter is slightly discolored compared to the rest of the filter. Subtle trapezoidal shapes surrounding the bacterial deposition can be seen pressed into the filter and these are due to support pillars intentionally fabricated in the insert base which press into the filter when the base is screwed into the insert main body. These shapes can be used to trivially locate the area of the filter where bacteria have been deposited even when no obvious discoloration is evident.

A comparison of Fig. 3(a) and 3(b) show that most of the region with the strongest bacterial signal is located inside the region where the cone was pressed into the filter, demonstrating that the cone was indeed effective at depositing bacterial cells onto a smaller region at the center of a filter. Measurement of non-zero bacterial signal outside of the limits set by the cone hole indicate that some cells moved laterally from underneath the cone hole during the centrifugation process. Elimination of this spreading would require the construction of a better temporary seal between the cone apex and the filter medium, perhaps by constructing a sharper apex that would press more firmly into the filter. Solutions to this spreading are hindered by the fact that the filter medium must be kept sterile and free from contamination prior to testing with LIBS. 

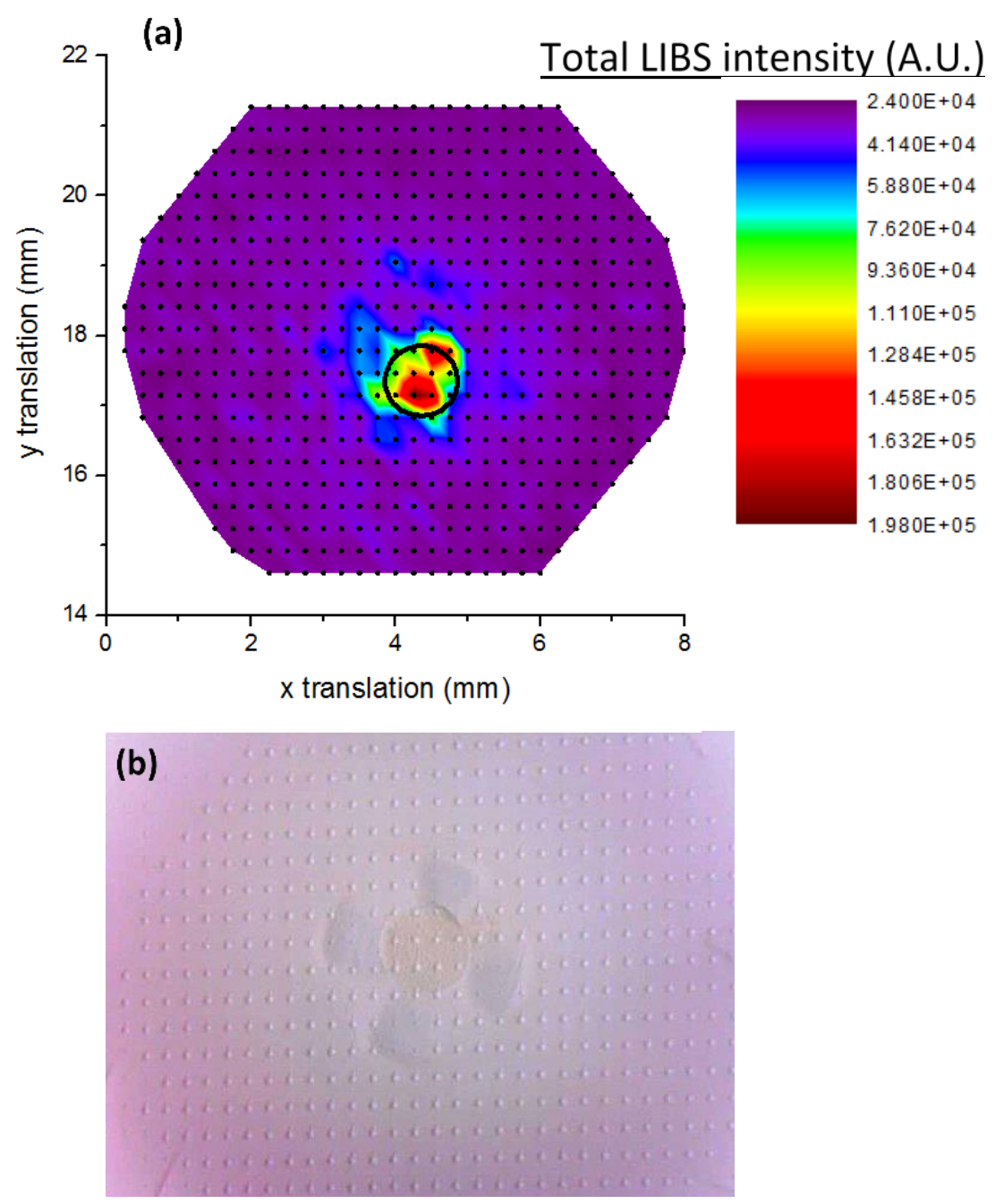

Fig. 3. (a) Color map depicting total LIBS intensity as a function of position on the filter. Each black dot represents the location of a data point corresponding to a single laser shot. The black circle indicates the approximate location of the $1 \mathrm{~mm}$ diameter cone hole on the filter. (b) Image of the filter after data acquisition. The array of craters due to the stepping of the LIBS laser can be seen. A slight discoloration due to the bacterial lawn is evident in the $1 \mathrm{~mm}$ diameter circular area at the center of the filter. The four trapezoidal indentations surrounding the bacterial lawn are due to support pillars constructed in the base of the centrifuge insert to hold the filter medium. The overall purplish tinge to the photograph is due to the camera and illumination.

\subsection{Limit of detection}

Nine different dilutions of a suspension of $E$. coli were prepared by serial dilution, and $30 \mu \mathrm{L}$ from each of the nine suspensions was deposited on filters using the metal cone with the conditions described in section 2.2. Representative volumes were tested with optical densitometry to determine the actual concentration. Each suspension was deposited on two separate filters for 
redundancy, and 20 single-shot LIBS spectra were collected in the region where the cone was pressed into the filter, resulting in a total of 40 LIBS spectra acquired for each suspension. The average and standard deviation of these 40 measurements were calculated. These data are presented in Fig. 4.

(a) All elements; all concentrations
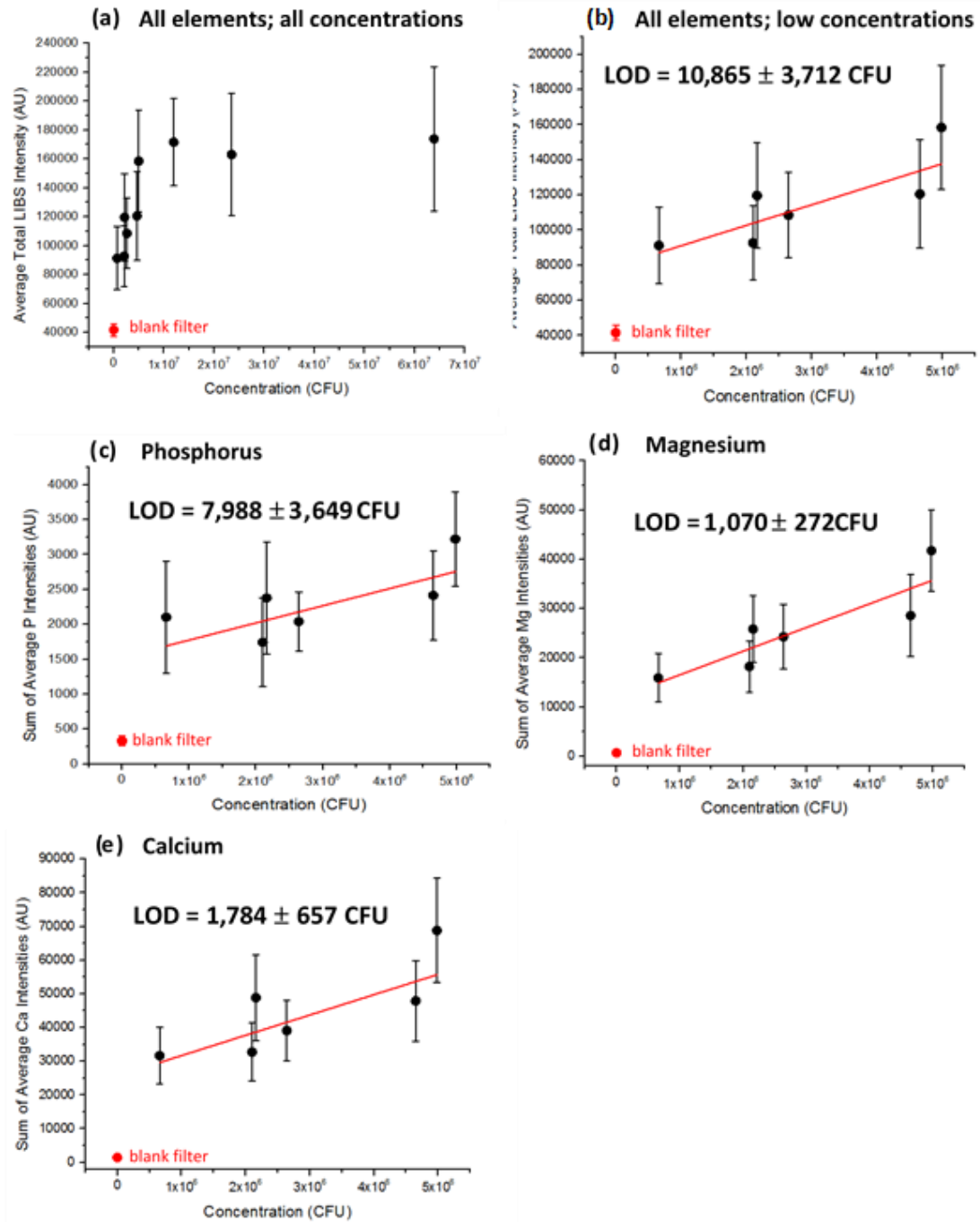

Fig. 4. (a) Plot of average total LIBS intensity as a function of CFU deposited on the filter for nine different initial concentrations and one blank filter. Error bars represent one standard deviation in the forty measurements. (b) Linear fit to the six concentrations which lie in the linear regime of the curve in (a). The LOD is the calculated $3 \sigma / m$ value. (c)-(e) Linear fits and calculated LODs for the six lowest concentrations when only the emission lines of phosphorus, magnesium, and calcium are utilized. The error bars for the blank filter are present, but are smaller than the data points.

Fig. 4(a) shows a calibration curve constructed from the average total LIBS intensity as a function of the total number of bacterial cells (in CFU) deposited on the filter for each of the nine suspensions along with a blank filter sample which contained no bacterial cells. It is important 
to note that this figure does not present a bacterial suspension concentration in units of $\mathrm{CFU} / \mathrm{mL}$ as described previously. Due to the way in which the suspension is tested in this work (i.e. centrifuged and passed through a filter) the volume of water was actually immaterial, and thus a concentration quantity is meaningless. For example, $1 \times 10^{7} \mathrm{CFU}$ suspended in both $1 \mathrm{~mL}$ and 2 $\mathrm{mL}$ of water would yield identical measured LIBS signals after being passed through the filter, but possessed different initial concentrations in units of $\mathrm{CFU} / \mathrm{mL}$. It is therefore much more practical to report the "concentration" merely as the quantity of bacteria, the total number of cells deposited on the filter, in units of CFU.

In Fig. 4(a) it can be seen that the sum of the background-subtracted intensities of the 19 emission lines measured to determine the number of bacterial cells deposited on the filter never actually reached zero when no bacteria were deposited, possessing an average total LIBS intensity around 40,000 arbitrary units due to the presence of strong carbon emission in the filter spectra, as well as smaller emissions from the trace elements. This is shown in the last column of Table 1 as the blank filter LIBS emission intensity. These data are labeled as "blank filter" in all the graphs of Fig. 4. Some amount of filter was unavoidably ablated with each shot and there was some contribution to the bacterial LIBS emission spectra from the filter for all concentrations of bacteria deposited on filter media. In principle, this quantity of observed filter emission could be subtracted from all summed intensities in an effort to "background subtract" the data. However the amount of filter media ablated was in general dependent on the thickness of bacterial deposition on the filter, and so the contribution to the spectra was not constant for the varying bacterial concentrations shown in Figure 4. Therefore the removal of the "blank filter" spectral contribution, which would be slightly different for every concentration and could not be determined, was not performed.

The average total LIBS intensity for each suspension was calculated by averaging the total LIBS intensities of the 40 spectra acquired for one suspension. It can be seen from Fig. 4(a) that a linear regime for this concentration curve exists for quantities of bacteria below approximately 1 
$x 10^{7} \mathrm{CFU}$. A linear fit was performed on the six data points in the linear dynamic range (excluding the blank filter data) and this is shown in Fig. 4(b). The errors in the measurements were included in the fit to calculate an uncertainty to the slope and the limit of detection (LOD). The LOD was determined using the standard $3 \sigma / m$ definition. The resulting LIBS bacterial LOD for bacteria deposited on filters using the metal cone was determined to be $10,865 \pm 3,712$ CFU per laser ablation event. This represents a five-fold improvement over the $\approx 50,000$ CFU per laser ablation event required for detection when cells were deposited with a custom-built circular metal jig as described in previous work [25] and the $\approx 90,000$ CFU per laser ablation event required for detection when deposited with the centrifuge tube insert device alone [29] as described previously.

The filter spectra possessed very low emission intensities for the trace elements of phosphorus, magnesium, and calcium relative to the bacterial emission spectra, as can be seen in Table 1. In addition the uncertainties in those intensities were small. Therefore concentration curves were constructed using only the emission lines measured for those specific elements and LOD measurements were constructed using these values. For each element, the intensities of all lines from that element were summed to calculate a single LIBS emission intensity for that element. Figs. 4(c-e) show the concentration curves for phosphorus, magnesium, and calcium, respectively. In each graph, the measured intensity for the blank filter is shown, with its appropriate uncertainty. In $4(\mathrm{~d}-\mathrm{e})$ the error bars in the filter measurements are smaller than the data points. Calculated limits of detection are given in Fig. 4 and are for phosphorus: 7,988 \pm 3,649 CFU; for magnesium: 1,070 $\pm 272 \mathrm{CFU}$; and for calcium: 1,784 $\pm 657 \mathrm{CFU}$. These values are approximately 50 times lower than our previously reported value. While these values represent a significant improvement in the LOD of the system, it is important to note that the limit of identification (LOI) will most likely require the measurement of many or most of the LIBS emission lines in the spectrum for efficient discrimination, and so is more likely to be on the order of the 10,865 CFU determined when utilizing all the lines observed in the spectrum. This is currently under investigation. 


\section{Conclusions}

A unique two-stage centrifuge tube insert device has been fabricated for rapid and convenient deposition of bacterial cells on disposable and inexpensive nitrocellulose filter media from liquid suspensions of arbitrary concentration. A custom metal cone utilized in conjunction with this insert allowed controlled deposition of an arbitrarily large or small number of bacterial cells within a circular area of approximately $1 \mathrm{~mm}$ diameter. This cone concentrated all the cells in a liquid suspension within a known area prior to LIBS testing regardless of the suspension's initial concentration. The bacteria deposited in this manner formed a uniform layer of bacterial cells which could be detected and identified on the basis of their LIBS spectra.

By testing suspensions with varying initial concentrations, an absolute limit of detection of 10,865 $\pm 3,712$ CFU per laser ablation event was calculated. Approximately 20 single-shot ablations could be performed upon each filter, although Fig. 3(b) shows clearly that a greater number of shots could be obtained by decreasing the translation distance between shots. However, for clinical applications it is not anticipated that a greater number of data points will be required, or even desired. In fact, it is most likely that only a single representative measurement will be needed for a diagnosis. To achieve this, all the spectra within the deposition region could be summed, reducing the LOD by greatly reducing the noise of the measurements (by the square root of the number of measurements so summed, in this case the square root of forty, or approximately 6.3). Conversely, fewer shots could be taken but the deposition area could be reduced, again reducing the LOD but this time by increasing the measured signal. As shown in Fig. 4(a), the signal is expected to grow linearly with increased bacterial cell numbers, but not indefinitely so it is not known to what extent this approach could lower the ultimate LOD. 
Lastly, while volumes of only $50 \mu \mathrm{L}$ were tested in this manuscript, the cone was designed to hold up to 20 times that volume, and in theory this technique could be scaled up further by constructing larger apparatus to fit inside larger centrifuges. If a significantly large volume of liquid could be passed through the cone-filter apparatus, the initial concentration could be quite low, perhaps in the 10 's of CFU/mL range, because the LOD of the test on the filter medium is independent of the initial concentration. It is dependent only on the absolute number of cells present in the liquid prior to centrifugation and filtration.

We have shown that this testing protocol can be done safely and rapidly (sample preparation steps required only minutes). In addition, since LIBS testing is performed on the surface of convenient and disposable filter media, there is no reason why a custom-fabricated benchtop apparatus could not be constructed to accept such filters which would require the operator to have little or no familiarity with the LIBS technique. Spectra so acquired could be identified autonomously by an appropriate chemometric model, providing almost real-time clinical diagnosis; although it is not yet known what the limit of identification is or what the sensitivity and specificity of the test are when using this apparatus. Nonetheless, due to the advantages just mentioned, this approach shows great promise for eventually being utilized in clinical or environmental microbiology laboratories.

\section{Acknowledgements}

The authors would like to gratefully acknowledge the financial support of a Natural Sciences and Engineering Research Council of Canada Discovery grant and RTI equipment grant as well as a Canada Foundation for Innovation - Ontario Innovation Fund infrastructure grant. In addition, 
portions of the work discussed in this review were supported by both the University of Windsor's

Outstanding Scholars program and NSERC USRA scholarships as well as a MITACS Globalink Research Internship.

[1] H.H. Telle, O. Samek, Biomedical applications of LIBS, in: A.W. Miziolek, V. Palleschi, I. Schechter (Eds.), Laser Induced Breakdown Spectroscopy. 1st ed., Cambridge University Press, Cambridge, 2006.pp. 282-313

[2] V.K. Singh, A.K. Rai, Prospects for laser-induced breakdown spectroscopy for biomedical applications: a review, Lasers Med. Sci. 26 (2011) 673-687. https://doi.org/10.1007/s10103-011-0921-2

[3] S.J. Rehse, H. Salimnia, A.W. Miziolek, Laser-induced breakdown spectroscopy (LIBS): an overview of recent progress and future potential for biomedical applications., J. Med. Eng. Technol. 36 (2012) 77-89.

https://doi.org/10.3109/03091902.2011.645946

[4] S.J. Rehse, Biomedical applications of LIBS, in: S. Musazzi, U. Perini (Eds.) Laser-Induced Breakdown Spectroscopy: Theory and Applications, Springer, Berlin, 2014, 457-488. https://doi.org/10.1007/978-3-64245085-3 17

[5] J.D. Hybl, G.A. Lithgow, S.G. Buckley, Laser-induced breakdown spectroscopy detection and classification of biological aerosols, Appl. Spectrosc. 57 (2003) 1207-1215.

[6] J. Diedrich, S.J. Rehse, S. Palchaudhuri, Escherichia coli identification and strain discrimination using nanosecond laser-induced breakdown spectroscopy, Appl. Phys. Lett. 90 (2007) 163901 1-3.

https://doi.org/10.1063/1.2723659

[7] S. Saari, S. Järvinen, T. Reponen, J. Mensah-Attipoe, P. Pasanen, J. Toivonen, J. Keskinen, Identification of single microbial particles using electro-dynamic balance assisted laser-induced breakdown and fluorescence spectroscopy, Aerosol Sci. Technol. 50 (2016) 126-132. https://doi.org/10.1080/02786826.2015.1134764 
[8] S. Manzoor, L. Ugena, J. Tornero-Lopéz, H. Martín, M. Molina, J.J. Camacho, J.O. Cáceres, Laser-induced breakdown spectroscopy for the discrimination of Candida strains, Talanta 155 (2016) 101-106. https://doi.org/10.1016/i.talanta.2016.04.030

[9] J.L. Gottfried, Discrimination of biological and chemical threat simulants in residue mixtures on multiple substrates, Anal. Bioanal. Chem. 400 (2011) 3289-3301. https://doi.org/10.1007/s00216-011-4746-4

[10] R.A. Multari, D.A. Cremers, M.L. Bostian, Use of laser-induced breakdown spectroscopy for the differentiation of pathogens and viruses on substrates, Appl. Opt. 51 (2012) B57-B64. https://doi.org/10.1364/AO.51.000B57

[11] S.J. Rehse, A Review of the Use of Laser-Induced Breakdown Spectroscopy for Bacterial Classification, Quantification, and Identification, Spectrochim. Acta B 154 (2019) 50-69.

https://doi.org/10.1016/j.sab.2019.02.005

[12] Q.I. Mohaidat, K. Sheikh, S. Palchaudhuri, S.J. Rehse, Pathogen identification with laser-induced breakdown spectroscopy: the effect of bacterial and biofluid specimen contamination, Appl. Opt. 51 (2012) B99-B107. https://doi.org/10.1364/AO.51.000B99

[13] G.R. Gamble, B. Park, S.-C. Yoon, K.C. Lawrence, Effect of sample preparation on the discrimination of bacterial isolates cultured in liquid nutrient media using laser-induced breakdown spectroscopy (LIBS), Appl. Spectrosc. 70 (2016) 494-504. https://doi.org/10.1177/0003702815626679

[14] D. Prochazka, M. Mazura, O. Samek, K. Rebrošová, P. Pořízka, J. Klus, P. Prochazková, J. Novotný, K. Novotný, J. Kaiser, Combination of laser-induced breakdown spectroscopy and Raman spectroscopy for multivariate classification of bacteria, Spectrochim. Acta B 139 (2018) 6-12. https://doi.org/10.1016/i.sab.2017.11.004

[15] J. Diedrich, S.J. Rehse, S. Palchaudhuri, Escherichia coli identification and strain discrimination using nanosecond laser-induced breakdown spectroscopy, Appl. Phys. Lett. 90 (2007) 163901 1-3.

https://doi.org/10.1063/1.2723659

[16] R. Multari, D.A. Cremers, J.M. Dupre, J.E. Gustafson, The use of laser-induced breakdown spectroscopy for distinguishing between bacterial pathogen species and strains, Appl. Spectrosc. 64 (2010) 750-759.

https://doi.org/10.1366/000370210791666183

[17] R.A. Multari, D.A. Cremers, M.L. Bostian, J.M. Durpr, J.E. Gustafson, Proof of principle for a real-time pathogen isolation media diagnostic: the use of laser-induced breakdown spectroscopy to discriminate bacterial pathogens and antimicrobial-resistant Staphylococcus aureus strains grown on blood agar, J. Pathog. 2013 (2013), 898106 111. https://doi.org/10.1155/2013/898106

[18] S. Manzoor, S. Moncayo, F. Navarro-Villoslada, J.A. Ayala, R. Izquierdo-Hornillos, F.J. Manuel de Villena, J.O. Caceres, Rapid identification and discrimination of bacterial strains by laser-induced breakdown spectroscopy and neural networks, Talanta 121 (2014) 65-70. https://doi.org/10.1016/i.talanta.2013.12.057

[19] S. Morel, N. Leone, P. Adam, J. Amouroux, Detection of bacteria by time-resolved laser-induced breakdown spectroscopy, Appl. Opt. 42 (2003) 6184-6191. https://doi.org/10.1364/A0.42.006184

[20] M. Baudelet, J. Yu, M. Bossu, J. Jovelet, J.-P. Wolf, T. Amodeo, E. Frejafon, P. Laloi, Discrimination of microbiological samples using femtosecond laser-induced breakdown spectroscopy, Appl. Phys. Lett. 89 (2006) 163903. https://doi.org/10.1063/1.2361270

[21] J. Cisewski, E. Snyder, J. Hannig, L. Oudejans, Support vector machine classification of suspect powders using laser-induced breakdown spectroscopy (LIBS) spectral data, J. Chemometrics 26 (2012) 143-149.

https://doi.org/10.1002/cem.2422

[22] A.K. Boardman, J. Campbell, H. Wirz, A. Sharon, A.F. Sauer-Budge, Rapid microbial sample preparation from blood using a novel concentration device, PLOS ONE 10 (2015) e0116837.

https://doi.org/10.1371/journal.pone.0116837

[23] P. Warnke, H. Frickmann, P. Ottl, A. Podbielski, Nasal screening for MRSA: different swabs - different results!, PLoS ONE 9 (2014) e111627. https://doi.org/10.1371/journal.pone.0111627 
[24] C. Barnett, C. Bell, K. Vig, C.A. Akpovo, L. Johnson, S. Pillai, S. Singh, Development of a LIBS assay for the detection of Salmonella enterica serovar Typhimurium from food, Anal. Bioanal. Chem. 400 (2011) 3323-3330. https://doi.org/10.1007/s00216-011-4844-3

[25] D.J. Malenfant, D.J. Gillies, S.J. Rehse, Bacterial suspensions deposited on microbiological filter material for rapid laser-induced breakdown spectroscopy identification, Appl. Spectrosc. 70 (2016) 485-493.

https://doi.org/10.1177/0003702815626673

[26] S.J. Rehse, Q.I. Mohaidat, S. Palchaudhuri, Towards the clinical application of laser-induced breakdown spectroscopy for rapid pathogen diagnosis: the effect of mixed cultures and sample dilution on bacterial identification, Appl. Opt. 49 (2010) C27-C35. https://doi.org/10.1364/AO.49.000C27

[27] W. Liao, Q. Lin, S. Xie, Y. He, Y. Tian, Y. Duan, A novel strategy for rapid detection of bacteria in water by the combination of three-dimensional surface-enhanced Raman scattering (3D SERS) and laser induced breakdown spectroscopy (LIBS), Anal. Chim. Acta 1043 (2018) 64-71. https://doi.org/10.1016/j.aca.2018.06.058

[28] R.A. Multari, D.A. Cremers, J.M. Dupre, J.E. Gustafson, Detection of biological contaminants on foods and food surfaces using laser-induced breakdown spectroscopy (LIBS), J. Agric. Food Chem. 61 (2013) 8687-8694.

https://doi.org/10.1021/jf4029317

[29] D.J. Malenfant, Influences on the Emissions of Bacterial Plasmas Generated through Nanosecond LaserInduced Breakdown Spectroscopy, University of Windsor, Windsor, 2016. https://scholar.uwindsor.ca/etd/5843

[30] A.E. Paulick, Development of Laser-Induced Breakdown Spectroscopy as a Rapid Diagnostic Tool for Bacterial Infection, University of Windsor, Windsor, 2018. https://scholar.uwindsor.ca/etd/7653/ 